\title{
IMPLEMENTATION OF CHORDAL RING NETWORK TOPOLOGY TO ENHANCE THE PERFORMANCE OF WIRELESS BROADBAND NETWORK
}

\author{
Nibras Habeeb Attrah \\ Department of Computer Science \\ Iraqi Commission for Computers and Informatics \\ Baghdad, Iraq \\ nibras.habeeb@muc.edu.iq \\ Ghassan H.Abdul-Majeed \\ Department of Scientific Affairs \\ University of Baghdad \\ Baghdad, Iraq \\ ghassan@uob.edu.iq \\ Mahmood Zaki Abdullah \\ Department of Computer Engineering \\ College of Engineering - Al-Mustansiriya University \\ Baghdad, Iraq \\ drmzaali@uomustnsiriyah.edu.iq
}

\begin{abstract}
The expansion of networks involved higher jump on the users utilizing the networks resources, which may require extra higher bandwidth. Due to the development of technology especially those folded under the Internet of Things (IoT), the new demand of higher data rate is been witnesses among the users. In order to feed the demand of users with high data rate, broadband networks are required where high data rate can be ensured for each user. Broadband networks can be established using optical network that carries the data through wide broadband. Areas such as rural and forests sides which are witnessing plenty of natural obstacles such as mountains, trees, seas, etc. are forming big challenge for propagating a cable (wire) or optical network.

Due to the limitations of the wire network, World Interoperability for Microwave Access (WiMAX) technology has been introduced as substitution for the broadband network. Such kind of alternative can be deployed through any geographical area without concerning on the wire paths. WiMAX preserved large coverage area and hence it may not suffer from the signal hand-off as in the case of another wireless network. In this project, Chordal Ring network topology is implemented to enhance the performance of wireless broadband network. With different routing protocols such as Destination Sequenced Distance Vector Protocol (DSDV) and Ad hoc On Demand Distance Vector (AODV), the network performance was examined for various Chordal Ring degree (e. g. fourth degree and fifth degree). Performance metrics such as number of transmitted packets, number of received packers, delivered packet rate (PDR), total number of drop packers (DP) and average queuing delay (been measured). Finally, the obtained results had been shown that fifth-degree Chordal Ring network is outperforming through DSDV routing protocol.
\end{abstract}

Keywords: AODV, DSDV, WiMAX, Chordal Ring Network, peer-to-peer, drop packers.

DOI: $10.21303 / 2461-4262.2021 .001734$

\section{Introduction}

Our society is becoming increasingly dependent on communication infrastructures, including broadband networks, and they are used for many aspects of our lives; Enterprises are relying on them for operating their businesses, contact with the public sector is increasingly based on self-service and digital platforms. and many services are (or are becoming) dependent on reliable high-capacity broadband connections, including services within health care, elderly care, education and home based workplaces. Wireless means transmitting signals using radio waves as the medium instead of wires [1]. Wireless technologies are used for tasks as simple as switching off the television or as complex as supplying the sales force with information from an automated enterprise application while in the field [2]. Now cordless keyboards and mice, pagers and digital and cellular phones have become part of our daily life. WiMAX systems are expected to deliver broadband access services to residential and enterprise customers in an economical way. Loosely, WiMAX is a standardized wireless version of Ethernet intended primarily as an alternative to wire technologies (such as Cable 
Modems) to provide broadband access to customer premises [3]. More strictly, WiMAX is an industry trade organization formed by leading communications, component, and equipment companies to promote and certify compatibility and interoperability of broadband wireless access equipment that conforms to the Institute of Electrical and Electronics Engineers, IEEE 802.16 standards [4].

WiMAX would operate similar to WiFi, but at higher speeds over greater distances and for a greater number of users. WiMAX has the ability to provide service even in areas that are difficult for wired infrastructure to reach and the ability to overcome the physical limitations of traditional wired infrastructure. WiMAX was formed in April 2001, in anticipation of the publication of the original 10-66 GHz Institute of Electrical and Electronics Engineers (IEEE 802.16) specifications. WiMAX is to 802.16 as the WIFI Alliance is to 802.11 . WiMAX is such an easy term that people tend to use it for the 802.16 standards and technology themselves, although strictly it applies only to systems that meet specific conformance criteria laid down by the WiMAX Forum $[5,6]$. The expansion of internet users and development of data technology has triggered big impact on the network development. The demand of high data rate is become insisting to provide the users with none interrupted services. Some applications are demanding data transmission in real-time such as video live steaming and television broadcasting, such applications might be supported with big bandwidth in order to tackle the delay problem [7]. The bandwidth requirements in such applications can be fulfilled by developing a robust infrastructure network [8]. The use of wire network and optical network provides the users with good data rate which serves the purpose of many applications e. g. video live broadcasting, web exploring, live video/audio chatting, file transfer, etc. In other word, broadband connection is required to maintain the series consistency of many network service providers and satisfy the users demand [9]. The propagation of wire network/fiber network in places alike hills areas and rural areas is not feasible. This challenge made the classical brad band connection in such areas not possible [10].

Optical networks are popular in their efficiency in terms of network reliability and large bandwidth, this type of networks with high expensive infrastructure. The utilization of optical network is taking place in accordance to the operational wavelength. The optical fiber can be operated using particular wavelength, another problem raised when that demand comes for another wavelength transmission rather than the standard wavelength. This need upgrading of the network infrastructure, which apply hefty burden on the cost [11]. Wireless networks are proposed to substitute the broadband network in the locations that wire network can be implemented. Data rate and bandwidth problem have remained even after deploying wireless networks. The radio channel is impacted by several environmental drawbacks such as the rain and electrometric fields. From the other hand, wireless network itself is suffering from spectrum limitation issue, which brings the bandwidth limitation once again.

\section{Materials and Methods}

In this section, let's discuss various topologies of networks, including ring, and chordal, with technical and theoretical details. Lastly enhanced proposed topology model will be presented in this section using NS2 simulator.

\section{1. Ring Topology}

Networks are designed for committing particular services for the nodes included in it. In order to ensure that commitment, networks are adopting particular topologies where the nodes are interfaced. The very basic network topology is termed as radial network which is about arranging the hosts' computers in straight line with the server and usually this topology is used in wire network for interference of printers and fax machines with the office network. Radial network using wire (coaxial) for its connection is susceptible for wire cut and hence services for the nodes after the cut will be dropped [12]. Another network topology has been created to enhance the rusticity of the connections as well as to increase the coverage of network to a distance more than one kilometer, this topology is called as ring network. Ring network topology is associated with both wire and wireless networks. It is more preferable in the context of optical communication where the optical fibers can be propagated for hundreds of kilometers with help of ring network [13]. Ring network, as in Fig. 1, is connecting $P$ nodes using a ring interfaces with diameter $D$ that stands for the distance from the ring's center reaching any terminal node. Ring is to be formed as each participated node is connected 
to only two nodes one in the lagging end and the other in the leading end. Standard ring network is not interrupted in-between (continuously propagated across all the nodes. If data request initiated from the node $p(1)$ and intended to reach node $p(10)$ in a ring network of 20 nodes; data request will reach the intended node by passing through all nodes intimidated in the path between $p(1)-p(10)$.

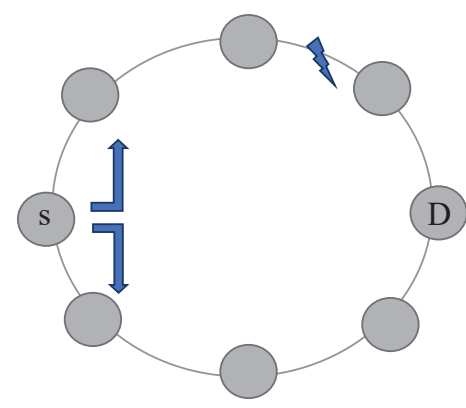

Fig. 1. Ring topology bi-directional transmission

However, ring network attributes can be listed here in:

a. Ring is forming a continuous pathway where request heading from one node to other node will be passing through all the nodes in the path.

b. Bidirectional transmission: the data transmitted from any source node either can be heading in the clockwise direction or on the anticlockwise direction reaching the destination node and considering the least pathway decided by the operative routing protocol governing the transmission process in the network.

c. Ring topology can cover wide geographical locations by using the repeaters or signal strength amplifiers.

d. The recent trends in the ring topology have made to prevent node isolation at the event of interfaces fail between any two nodes. This development is inspired by using the ring counter-rotation. This attribute allows the failed request to reach the destination node by changing the direction away from the faulty route as in Fig. 1.

\section{2. Chordal Ring}

Ring network as well as other networks is susceptible for several performance degradations which can be listed in the following points:

a. In wire network: The interfaces between the nodes are susceptible of cutting of fault at any time since the wire are commonly propagated along wide distances and they are exposed to wind, rain and temperature fluctuations. So-to-say, wire connection can be cut and hence, the network services to the further nodes could be interrupted [14].

b. In optical fiber network, requirement of wavelength varying might be raised and that required upgrading the optical cable in order to permit different wavelength to be travelled. The cost associated with cable upgrading is very high.

c. In wireless networks: channel allocation is problematic since the unlicensed users are creating a challenge for the networks planners. However, band and spectrum issue may not allow creating extra channels (bandwidth) so; it will create another problem for data transmission.

In order to address the aforementioned challenges, Chordal Ring networks are proposed, it simply works by propagating a standalone link (could be wire or wireless) between the node pairs in order to provide alternative pathway to transfer the data between those pairs. Chordal Ring network is providing alternative pathway for the data that not compatible with the original channel conditions. This data may need extra bandwidth over that in the main wire as the case in optical channel Fig. 2.

Fig. 2 demonstrated the transmission of two different bandwidths from the node A to node $\mathrm{B}$, the first bandwidth (wide band) is being transmitted through the channel labeled (bandwidth 1) while the other bandwidth (narrow band) is being transmitted over the Chordal link. In this example, channel was established between the both terminals and hence transmission between both terminals are governing by the channel specifications e.g. bandwidth, wavelength, etc. $[15,16]$. 


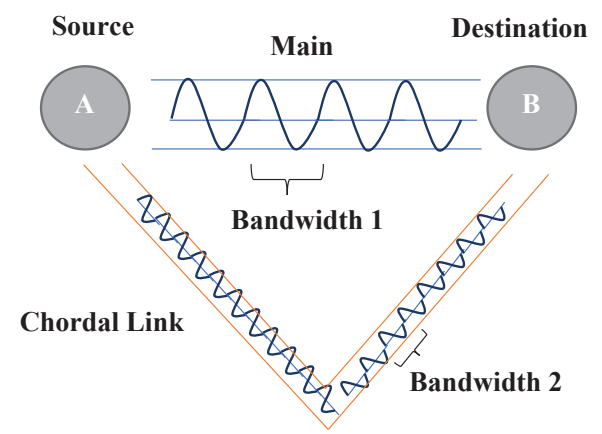

Fig. 2. Chordal Ring model transmission style

However, if another requirement was raised likely to exchange data with different bandwidth requirements, that may require upgrading of the existing channel which is not an easy task. However, the alternative link (Chordal link) may solve this problem by providing the channel requirement for that particular data and ensure delivery of data to the destination node.

\section{3. Development of model}

Chordal ring topology is proposed as underlying network topology for the WiMAX, however, model is being fabricated so that, different routing protocols are used e.g. AODV and DSDV. Model is developed using network simulator version II (NS2) and model is made using of ten nodes each node is participating the ring topology where the topology radius is one-hundred kilometer. Two different possibilities of Chordal degree are used likely fourth and fifth degree of Chordal Ring. The model is tested by varying the topology degree as well as the routing protocol. Results are determined in each case using the following performance metrics:

a. Number of transmitted packets (Tx packets): which reflects the total number of packets that being transmitted in the network from the source node to the destination nodes.

b. Number of received packets (Rx packets): which reflects the total number of packets that being received in the network from the source node by the destination nodes.

c. Packet delivery rate (PDR): the ratio of the total delivered packets in the network to the total transmitted packets determined for all the network nodes.

d. Dropped packets (DP): total number of packets that have been dropped while it transmits from the source node into destination nodes.

e. Latency: the average time (in seconds) taken by the packet to deliver from the source node, calculated after the destination node receives the packet and acknowledge the reception of the same.

\section{3. 1. Plain Vs. Chordal Ring}

The plain ring topology based IEEE 802.16 (WiMAX) network is made with ten nodes and diameter of $100 \mathrm{~km}$ as shown in Fig. 3.

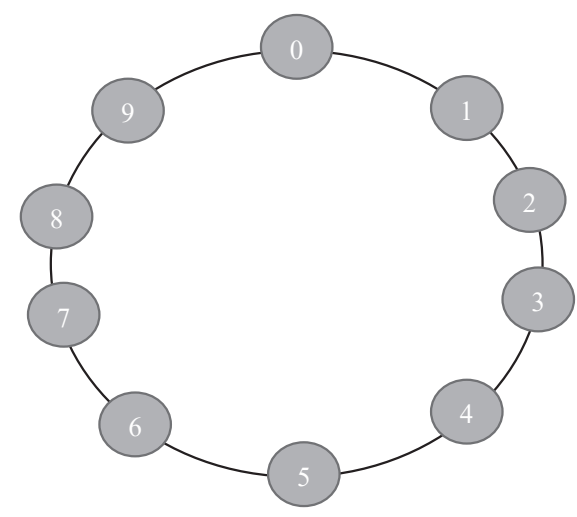

Fig. 3. Ring topology (plain) without chords (first model) 
The traffic will begin from node ZERO and will propagate through all nodes reaching again to the node ZERO. Each node will wirelessly transmit its payload to the next node in the ring and similarly the further nodes will follow the same transmission regime. Two routing protocols are used respectively namely AODV and DSDV. Network performance is being studied using the aforementioned performance metrics. Fig. 5, 7 demonstrates the topologies of Chordal degree 4 and 5 respectively. The NS2 models of the proposed Chordal rings are presented in Fig. 4, 6. Fig. 8 explains the ring topology based on fifth degree chords using Ns2 simulated model.

The Chordal Ring stands for an enlarged ring, or a circulated graph with a chord of length 1. Properly, it can be well-defined as a result of the pair $(n, L)$ in which $n$ symbolizes the amount of nodes of the ring, and $L$ stands for the set of chords.

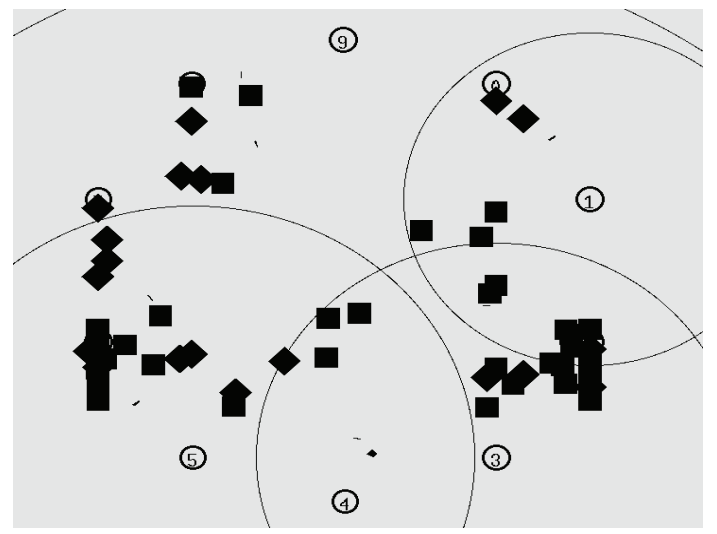

Fig. 4. NS2 simulated ring topology (plain) without chords (first model)

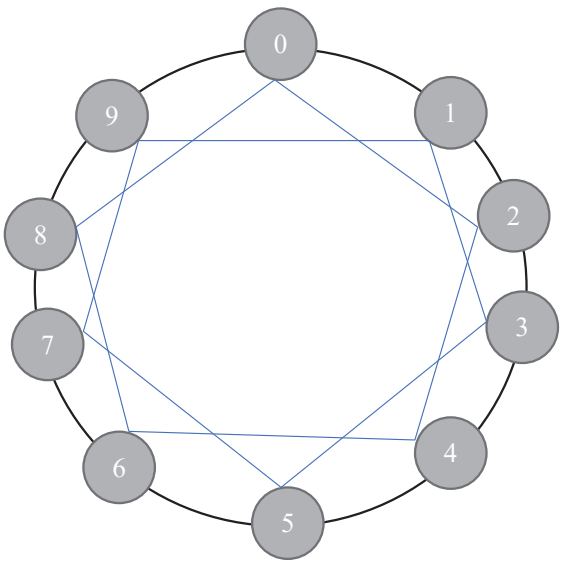

Fig. 5. Ring topology with 4 th degree chords

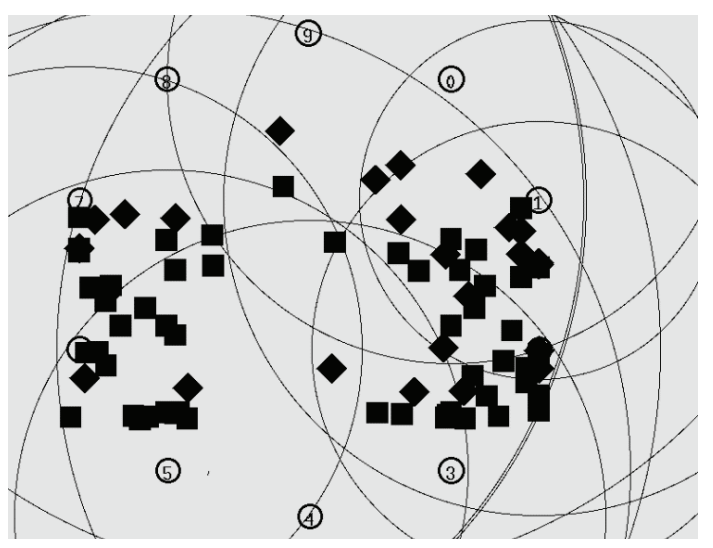

Fig. 6. Ring topology with 4th degree chords (NS2 simulated model) 


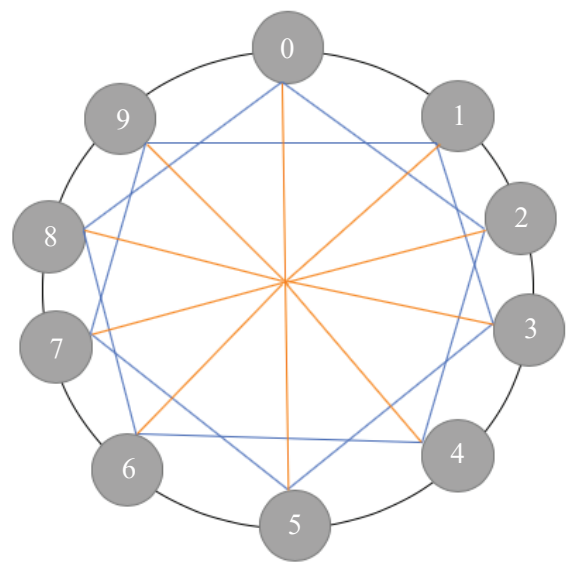

Fig. 7. Ring topology with fifth degree chords

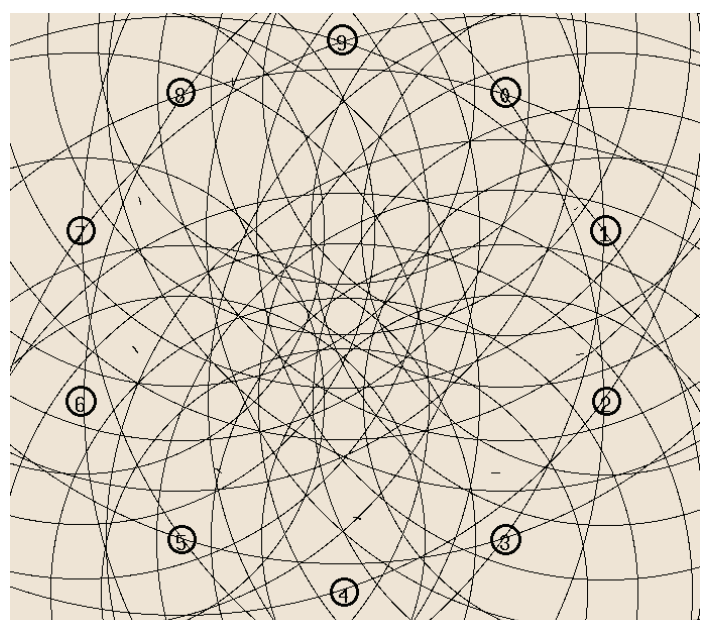

Fig. 8. Ring topology with fifth degree chords (Ns2 simulated model)

\section{Results and Discussion}

Three models are implemented in network simulator II according to the settings mentioned at the above section. The performance of the simulated models is observed under two different routing protocols (AODV and DSDV).

The results are shown in Table 1 that performance of network with DSDV protocol is better than in AODV protocol. Taking in consideration different network topologies are being in use, results revealed that, the bigger chordal degree the more losses in terms of time delay.

Tables 1, 2 demonstrate proposed models performance in case of AODV and DSDV routing protocol respectively.

Wireless broadband and optical network have been for long time undergoing extensive research activities. In some locations such as agriculture areas, propagating the optical network is not feasible due to the natural obstacles. Furthermore, optical or any wire network is susceptible for cutting where the cost of maintenance will be far costly than normal network. Alternatively, wireless broadband network is proposed in this project to replace the traditional optical network. Connecting of fixed wireless broadband network may solve the data rate problem and provide easy to access network that does not affect by the natural obstacles alike trees or mountains. In order to ensure smooth functionality of wireless broadband network, Chordal Ring network topology is proposed. Wireless data rate and speed is directly impacted by the environmental conditions such as rain and humidity.

So, the proposed Chordal Ring broadband wireless network is expected to tackle those issues and provide a stable network performance. The existence of Chordal wireless ring is to prevent the network congestion and to substitute the bandwidth shortage. The fourth and fifth 
degree (fourth degree and fifth degree) ring topology based IEEE 802.16 (WiMAX) network is made with ten nodes and diameter of $100 \mathrm{~km}$.

Table 1

AODV routing protocol performance at the all proposed models

\begin{tabular}{cccccc}
\hline Network & $\boldsymbol{T} \boldsymbol{x}$ packets & $\boldsymbol{R} \boldsymbol{x}$ packets & PDR \% & DP & Latency $(\mathbf{s})$ \\
\hline Ring & 9000 & 7008 & 77.86667 & 1992 & 3.45 \\
$4^{\text {th }}$ degree & 27000 & 6250 & 69.44444 & 2750 & 11.1731 \\
$5^{\text {th }}$ degree & 36000 & 4514 & 50.15556 & 4486 & 14.1319
\end{tabular}

Table 2

DSDV routing protocol performance at the all proposed models

\begin{tabular}{cccccc}
\hline Network & $\boldsymbol{T} \boldsymbol{x}$ packets & $\boldsymbol{R} \boldsymbol{x}$ packets & PDR \% & DP & Latency (s) \\
\hline Ring & 9000 & 7127 & 79.18889 & 1873 & 3.68417 \\
$4^{\text {th }}$ degree & 27000 & 7185 & 79.83333 & 1815 & 10.2749 \\
$5^{\text {th }}$ degree & 36000 & 7216 & 80.17778 & 1784 & 11.1947
\end{tabular}

Consequences revealed that Chordal Ring network may apply queuing delay at the time of packets transmission. As the degree of chords is increased, the latency will be increased. Form the other hand, the packet delivery ratio is increased by increasing the degree of the Choral Ring. Consequences have shown that DSDV protocol is outperformed the AODV protocol.

It is applicable to implement different suggestions for improving the proposed management system. The suggestions are:

1. Proposing a design for a large number of nodes of chordal ring networks, like 8 nodes nets or greater than that.

2. Strengthening the chordal ring system in terms of security and adding algorithms to maintain the security of information.

3. Improving the packet delivery of information through chordal ring by proposing a new approach and algorithms for updating routing tables.

4. Proposing a new scheme for multicasting based on no nodes or links failure, future research may deal with nodes or links failure in chordal ring networks.

5. Performance optimization can be done by Genetic Algorithm (GA). It is a metaheuristic inspired by the natural selection process belonging to the wider evolutionary algorithm (EA). Genetically-inspired operators such as mutation, crossover, and selection are often used to provide high-quality solutions for optimization and research problems [17].

\section{Conclusion}

1. Connecting of fixed wireless broadband network may solve the data rate problem and provide easy to access network that does not affect by the natural obstacles alike trees or mountains.

2. In order to ensure smooth functionality of wireless broadband network, chordal ring network topology is proposed.

3. Wireless data rate and speed is directly impacted by the environmental conditions such as rain and humidity. So, the proposed chordal ring broadband wireless network is expected to tackle those issues and provide a stable network performance.

4. The existence of chordal wireless ring is to prevent the network congestion and to substitute the bandwidth shortage. The third, fourth and fifth degree $(D=3, D=4$ and $D=5)$ ring topology is made for different and diameter and zone area.

5. Obtained results reveal that chordal ring network may apply queuing delay at the time of packets transmission. As the degree of chords is increased, the latency will be increased.

6. The packet delivery ratio is increased by increasing the degree of the choral ring.

7. Obtained results show that DSDV protocol is outperformed over the AODV protocol. 


\section{References}

[1] Pedersen, J. M., Zabłudowski, A., Gutierrez, J., Ledziński, D., Dubalski, B. (2012). A Survey on Chordal Rings, N2R and Other Related Topologies. Image Processing \& Communications, 17 (3), 63-70. doi: https://doi.org/10.2478/v10248-012-0022-2

[2] Chen, Y., Shen, H., Zhang, H. (2006). Embedding Hypercube Communications on Optical Chordal Ring Networks. Proceedings. 2006 31st IEEE Conference on Local Computer Networks. doi: https://doi.org/10.1109/lcn.2006.322132

[3] Bujnowski, S., Dubalski, B., Zabludowski, A., Ledzinski, D., Riaz, M. T., Pedersen, J. M. (2010). Evaluation of modified degree 5 chordal rings for network topologies. 2010 Australasian Telecommunication Networks and Applications Conference. doi: https://doi.org/10.1109/atnac.2010.5680257

[4] Dubalski, B., Zabludowski, A., Bujnowski, S., Pedersen, J. M. (2008). Comparison of Modified Chordal Rings Fourth Degree to Chordal Rings Sixth Degree. 2008 50th International Symposium ELMAR, 597-600.

[5] Abbas, A. (2006). Broadcasting Algorithm on Large Chordal Ring of Degree Six Networks. 2006 2nd International Conference on Information \& Communication Technologies. doi: https://doi.org/10.1109/ictta.2006.1684917

[6] Kitani, T., Funabiki, N., Higashino, T. (2004). A proposal of hierarchical chordal ring network topology for WDM networks. Proceedings. 2004 12th IEEE International Conference on Networks (ICON 2004) (IEEE Cat. No. 04EX955). doi: https:// doi.org/10.1109/icon.2004.1409241

[7] Parhami, B., Kwai, D.-M. (1999). Periodically regular chordal rings. IEEE Transactions on Parallel and Distributed Systems, 10 (6), 658-672. doi: https://doi.org/10.1109/71.774913

[8] Fang, J.-F., Hsiao, J.-Y., Tang, C.-Y. (1998). Embedding meshes and TORUS networks onto degree-four chordal rings. IEE Proceedings - Computers and Digital Techniques, 145 (2), 73. doi: https://doi.org/10.1049/ip-cdt:19981903

[9] Mans, B. (1999). On the interval routing of chordal rings. Proceedings Fourth International Symposium on Parallel Architectures, Algorithms, and Networks (I-SPAN'99). doi: https://doi.org/10.1109/ispan.1999.778911

[10] Pedersen, J. M., Riaz, T. M., Dubalski, B., Madsen, O. B. (2008). A Comparison of Network Planning Strategies. 2008 10th International Conference on Advanced Communication Technology. doi: https://doi.org/10.1109/icact.2008.4493857

[11] Zabludowski, A., Dubalski, B., Bujnowski, S., Riaz, T. (2008). Transport Layers for Backbone and Access IP Networks. 2008 50th International Symposium ELMAR.

[12] Fang, L., Wilson, W. H. Study of Sequence Processing on Neural Networks.

[13] Rodrigues, J. J. P. C., Freire, M. M., Lorenz, P. (2005). One-Way Resource Reservation Protocols for IP Over Optical Burst Switched Mesh Networks. 2005 Systems Communications (ICW'05, ICHSN'05, ICMCS'05, SENET'05). doi: https://doi.org/ 10.1109/icw.2005.62

[14] Qiao, C., Yoo, M. (1999). Optical burst switching (OBS) - A new paradigm for an optical Internet. Journal of High Speed Networks, 8 (1), 69-84.

[15] Turner, J. S. (1999). Terabit burst switching. Journal of High Speed Networks, 8 (1), 3-16.

[16] Baldine, I., Rouskas, G. N., Perros, H. G., Stevenson, D. (2002). JumpStart: a just-in-time signaling architecture for WDM burst-switched networks. IEEE Communications Magazine, 40 (2), 82-89. doi: https://doi.org/10.1109/35.983912

[17] Mezaal, Y. S., Abdulkareem, S. F. (2017). Affine cipher cryptanalysis using genetic algorithms. JP Journal of Algebra, Number Theory and Applications, 39 (5), 785-802. doi: https://doi.org/10.17654/nt039050785

Received date 30.10 .2020

Accepted date 15.03.2021

Published date 31.03.2021
(C) The Author(s) 2021

This is an open access article under the CC BY license (http://creativecommons.org/licenses/by/4.0).

How to cite: Attrah, N. H, Abdul-Majeed, G. H., Abdullah, M. Z. (2021). Implementation of chordal ring network topology to enhance the performance of wireless broadband network. EUREKA: Physics and Engineering, 2, 11-18. doi: https://doi.org/10.21303/ 2461-4262.2021.001734 